\title{
Parameters Contributing to Efficient Ion Generation in Aerosol MALDI Mass Spectrometry
}

\author{
Erica L. McJimpsey, ${ }^{\mathrm{a}, \mathrm{b}}$ William M. Jackson, ${ }^{\mathrm{a}}$ Carlito B. Lebrilla, ${ }^{\mathrm{a}}$ \\ Herbert Tobias, ${ }^{\mathrm{b}}$ Michael J. Bogan, ${ }^{\mathrm{b}}$ Eric E. Gard, ${ }^{\mathrm{b}}$ Matthias Frank, \\ and Paul T. Steele \\ a University of California, Davis, Davis, California, USA \\ ${ }^{\mathrm{b}}$ Lawrence Livermore National Laboratory, Livermore, California, USA
}

The Bioaerosol Mass Spectrometry (BAMS) system was developed for the real-time detection and identification of biological aerosols using laser desorption ionization. Greater differentiation of particle types is desired; consequently MALDI techniques are being investigated. The small sample size $\left(\sim 1 \mu \mathrm{m}^{3}\right)$, lack of substrate, and ability to simultaneously monitor both positive and negative ions provide a unique opportunity to gain new insight into the MALDI process. Several parameters known to influence MALDI molecular ion yield and formation are investigated here in the single particle phase. A comparative study of five matrices $(2,6-$ dihydroxyacetophenone, 2,5-dihydroxybenzoic acid, $\alpha$-cyano-4-hydroxycinnamic acid, ferulic acid, and sinapinic acid) with a single analyte (angiotensin I) is presented and reveals effects of matrix selection, matrix-to-analyte molar ratio, and aerosol particle diameter. The strongest analyte ion signal is found at a matrix-to-analyte molar ratio of 100:1. At this ratio, the matrices yielding the least and greatest analyte molecular ion formation are ferulic acid and $\alpha$-cyano4-hydroxycinnamic acid, respectively. Additionally, a significant positive correlation is found between aerodynamic particle diameter and analyte molecular ion yield for all matrices. SEM imaging of select aerosol particle types reveals interesting surface morphology and structure. (J Am Soc Mass Spectrom 2008, 19, 315-324) (c) 2008 American Society for Mass Spectrometry

$\mathrm{T}$ The Bioaerosol Mass Spectrometry (BAMS) system was developed for national security and public health applications including the real-time, in situ detection of airborne pathogens [1] and biowarfare agents $[2,3]$. It has the ability to distinguish Bacillus spores from vegetative cells on a particle by particle basis using ion peaks under $\mathrm{m} / \mathrm{z} 300$ [4], and has demonstrated preliminary species level differentiation of individual Bacillus spores using low mass biomarker peaks $[5,6]$. In spite of this success, the production, detection, and identification of higher-mass taxonomical biomarker peaks is strongly desired because it would enable more robust differentiation of a broader range of bacterial species. With this goal in focus, early investigations into single particle matrix-assisted laser desorption/ionization (MALDI) techniques are described here using standard peptide particles. This avoids the inherent variability and indefinite composition of biological particles, which obscure the phenomena of present interest.

Address reprint requests to Dr. E. L. McJimpsey, Department of Chemistry, University of California, Davis, One Shields Ave., Davis, CA 95616, USA. E-mail: mcjimpsey2@llnl.gov
Single particle mass spectrometry has now been practiced for more than 20 years. In 1984, Sinha et al. first described a method for the "laser-induced volatilization and ionization of individual microscopic particles on a continuous, real-time basis" [7]. In this first apparatus, full spectra could not be acquired from single particles due to the use of a quadrupole mass spectrometer, and only monodisperse aerosols could be efficiency analyzed. In 1988, Marijnissen et al. proposed the use of a time-of-flight mass spectrometer (TOFMS) [8], but McKeown et al. in 1991 were the first to build and describe an on-line system [9]. Prather et al. added aerodynamic sizing to a TOFMS to enable the accurate sizing and efficient analysis of polydisperse aerosols in 1994 [10]. In 1996, Hinz et al. effectively doubled the amount of information obtained from individual particles by utilizing a dual polarity TOFMS [11]. The Prather group would follow suit and in 1997 describe portable systems employing aerodynamic sizing and a dual-polarity reflectron TOFMS. These later systems built by Gard et al. [12] would eventually evolve into the BAMS system at Lawrence Livermore National Laboratory [13]. The most current BAMS systems now efficiently focus aerosols spanning the entire respirable 
size range $(1-10 \mu \mathrm{m})$, track particles at rates of 10,000 per second or more, probe single particle laser-induced fluorescence at rates of 1000 per second or more, and even measure single particle charge in addition to acquiring dual-polarity mass spectra. This is coupled with significant software and firmware developments enabling the sensitive, sub-minute detection and identification of various aerosol threats.

Such a brief outline of early hardware advances leading, in part, to the development of the BAMS system glosses over many significant developments and contributions made by other groups. In 1994, Dale et al., for example, began a distinct line of instrument development by creating a system using a quadrupole ion trap [14]. In that same year, Murhpy et al. described a more conventional instrument $[15,16]$ and in 1998 conducted unique studies sampling particles at 5 to $19 \mathrm{~km}$ from the nose of an airplane [17]. These and other important works [18, 19] are, however, reviewed elsewhere [20, 21]. One very important development that requires mention here, though, is the application of bulk MALDI techniques to single particle mass spectrometry.

Over the past two decades, MALDI has developed as a powerful tool for the analysis of large mass biomolecules [22-24]. For instance, the ability to differentiate spore species in bulk using small acid soluble proteins (SASPs) has been shown [25]. In such experiments, carefully prepared samples are typically placed on a sample plate, dried, introduced into vacuum, and then irradiated. This is not efficacious, however, for the type of high throughput single particle analysis desired for real-time threat particle detection.

Aerosol MALDI allows single particles to be rapidly analyzed. It has not been as well studied as bulk MALDI, but it does provide a unique opportunity for fundamental research into the MALDI process. The analysis of aerosol particles containing analyte and matrix was first reported in 1993 by Murray and coworkers [26], who would go on to publish numerous papers on the subject [27-34]. In 1996, however, Mansoori et al. were the first to analyze such particles individually in a single particle TOFMS [35]. In 2005, Harris et al. described aerosol MALDI in an ion trap mass spectrometer [36]. A number of groups have analyzed proteins, but in 2000 , Stowers et al. were the first to analyze Bacillus spores $[37,38]$. Regardless of the analyte, aerosol MALDI differs from classical bulk MALDI in a number of important ways. It lacks a sample probe and involves a sample volume typically 2 to 5 orders of magnitude smaller than that probed in bulk MALDI. Differences in optimal laser fluence [33], matrix-to-analyte molar ratio [27], choice of matrix [31], as well as other experimental parameters are thus expected and have been observed. They will be examined here afresh, however, using a standard peptide with various matrices. The ability to observe both positive and negative ions simultaneously provides a novel opportunity to gain new insights into these phenomena. Such investigations complement other on- going investigations of both aerosol and bulk MALDI and help elucidate the common underlying processes involved.

Ultimately, aerosol MALDI may enable the high level of specificity desired for the BAMS system by providing high sensitivity and a higher operational mass range. Employing MALDI techniques with the BAMS system has already demonstrated zeptomole detection of peptides [39]. Future studies will likely progress to larger peptides, toxins (or surrogates), viruses, spores, and cells. In the end, more robust mass spectral identification of bioterror agents is expected.

\section{Experimental}

\section{Instrumentation}

All experiments were performed using a BAMS system with a dual polarity linear time-of-flight mass spectrometer [40]. The BAMS system analyzes particles of varying chemical composition, including aerosols of a nonbiological nature. In this particular system, particles are entrained in air and sampled into the instrument via a converging nozzle at atmospheric pressure. The particles exit the nozzle at a pressure of 2 torr. This pressure differential causes the sampled gas to undergo a supersonic expansion during which the smaller entrained particles are accelerated to a higher terminal velocity than the larger particles. The expansion also causes the particles to become focused into a beam, which subsequently passes through two stages of differential pumping, at pressures of $10^{-1}$ and $10^{-4}$ torr, respectively. Individual particles in the focused particle stream are then tracked by three continuous wave $(\mathrm{CW})$ diode scattering lasers. The detected scattered light pulses are generated from individual particles passing through the laser beams and then sent to a timing circuit, which measures the time for each particle to pass from the first to the last scattering laser. Since the distance between the lasers is known, the terminal velocity of each particle is easily determined. Using this terminal velocity, an algorithm calculates the aerodynamic diameter of each particle. A particle's velocity combined with the exact time it passes through the last scattering laser provide the necessary timing to appropriately fire a $355 \mathrm{~nm}$ Nd:YAG desorption/ionization (DI) laser (Big Sky Laser Technologies, Inc. Ultra, Bozeman, MT) as the particle reaches the center of the mass spectrometer ion source. The DI laser, which is aligned perpendicular to both the particle beam and the axis of the spectrometers, produces $7 \mathrm{~ns}$ pulses that are flattopped and focused to a spot size of $380 \mu \mathrm{m}$ [41]. Delayed extraction is then used to simultaneously accelerate both positive and negative ions into the opposing linear time-of-flight mass spectrometers, each configured with ion guiding capabilities and identically biased, MCP-based detectors. The delay time, or the time in which ions are allowed to propagate freely before extraction from the source, was optimized for peak resolution at $320 \mathrm{~ns}$. The guide wire focuses divergent ion trajectories by creating an inwardly attractive radial elec- 
tric field within each cylindrical flight tube and was not pulsed. A dual polarity mass spectrum is thus obtained for each individual particle analyzed.

The raw spectral data collected from each particle consisted of 131,000 data points per polarity, sampled at $500 \mathrm{MHz}$ and stored directly to a computer. The stored data were convolved with a Gaussian smoothing function, having a full width at half maximum of $10 \mathrm{~ns}$, to filter out high-frequency noise and increase the signal to noise ratio. A baseline offset was then subtracted before calibration and subsequent analysis.

\section{Materials}

The matrices 3'5-dimethoxy-4-hydroxycinnamic acid (sinapinic acid, SA), 4-hydroxy-3-methoxycinnamic acid (ferulic acid, FA), 2'6'-dihydroxyacetophenone (DHAP), 2'5'-dihydroxybenzoic acid (DHB), and $\alpha$-cyano-4hydroxycinnamic acid (HCCA) were obtained from Sigma Chemical Co. (Steinham, Belgium) and used without further purification. Angiotensin I was purchased from SynPep (Dublin, CA). Ethanol solvent (HPLC grade) was purchased from Sigma. Trifluoroacetic acid (HPLC grade) was purchased from J. T. Baker, Phillipsburg, NJ. All were used without further purification.

\section{Sample Preparation}

Separate solutions of the analyte, angiotensin I, with each of the matrices SA, FA, DHAP, DHB, and HCCA were prepared and investigated. All solutions were prepared at a total concentration of $7.14 \times 10^{-4} \mathrm{M}$ but with varying matrix-to-analyte molar ratios of $50 \mathrm{~K}$ to 1 , $10 \mathrm{~K}$ to $1,1 \mathrm{~K}$ to 1,100 to 1 , and 10 to 1 . The solvent system consisted of $\mathrm{H}_{2} \mathrm{O}$ /ethanol (1:1) and $0.1 \%$ TFA by volume. Aerosol droplets were generated using a collision nebulizer from a solution containing the solvent system, analyte, and matrix. The nebulized, polydisperse droplets were passed through a silica drying column containing a perforated cylinder, $50 \mathrm{~cm}$ long and $1 \mathrm{~cm}$ in diameter, surrounded by silica particles at a flow rate of $\sim 1.5 \mathrm{~L} / \mathrm{min}$. The dried particles were carried by a stream of air through a tube and sampled into the instrument.

\section{Results and Discussion}

Numerous parameters are known to affect mass spectral quality in both aerosol and bulk MALDI mass spectrometry. The factors that have the greatest effect include laser fluence, type of matrix, and matrix-toanalyte molar ratio. These factors have previously been studied for a variety of reasons [13, 27, 31]. In this work, these parameters, along with the size of the aerosol particles, are examined specifically to determine their effect upon molecular analyte ion signal. This is prudent given the goal of high sensitivity and extended mass range. The authors have investigated laser fluence in various contexts before $[13,41]$, so it is discussed only briefly here and grouped with a description of observed ion peaks. More substantial examinations of the other parameters follow.

\section{Laser Fluence}

Laser fluence is a well known and important factor influencing ion generation in both bulk [42] and single particle $[13,43]$ mass spectrometry. The DI laser fluence was optimized for maximal analyte ion signal intensity in dual polarity using the matrix/analyte solution of SA and angiotensin. Ion signal, in this case, was defined as the integrated signal from the multiple analyte molecular ions observed. Based on the optimization, all spectra were consequently acquired at a laser pulse energy of $0.30 \mathrm{~mJ}$, a fluence of $260 \mathrm{~mJ} / \mathrm{cm}^{2}$ and an irradiance of $3.8 \times 10^{7} \mathrm{~W} / \mathrm{cm}^{2}$. Optimal bulk MALDI fluences range between $1 \mathrm{~mJ} / \mathrm{cm}^{2}$ and $50 \mathrm{~mJ} / \mathrm{cm}^{2}$ [42]. The high fluence required here compared with bulk was not unexpected. It has been suggested previously that residual solvent in the particles helps dissipate excess absorbed laser energy [28, 33]. Other processes are likely involved as well. In bulk, as the area of the laser-sample interaction region decreases, the apparent threshold fluence increases [42]. Thus, in single particle analysis, where the laser sample interaction region (i.e., the particle) is typically orders of magnitude smaller than in bulk, higher fluences should be expected irrespective of any solvent effect. In the discussion of matrix-to-analyte ratio, a specific process potentially contributing to this size dependence is suggested.

The selected single particle spectra in Figure 1 for DHAP and HCCA display many of the observed angiotensin molecular ion peaks. A single particle spectrum represents the ions created from a single micron-sized particle. Fragmentation of analyte molecular ions was not observed with any of the matrices studied. The analyte molecular ions observed in the positive polarity included the protonated $[\mathrm{A}+\mathrm{H}]^{+}$, sodiated $[\mathrm{A}+\mathrm{Na}]^{+}$, potassiated $[\mathrm{A}+\mathrm{K}]^{+}$, and deprotonated but sodiated and potassiated (deprotonated $\mathrm{NaK}$ ) $[\mathrm{A}+\mathrm{Na}+\mathrm{K}-$ $\mathrm{H}^{+}$species, producing peaks at $1298,1320,1336$, and $1358 \mathrm{~m} / \mathrm{z}$, respectively. In the negative polarity, the angiotensin molecular ion species included a deprotonated $[\mathrm{A}-\mathrm{H}]^{-}$peak at $1296 \mathrm{~m} / \mathrm{z}$ and a sodiated but doubly deprotonated peak $[\mathrm{A}+\mathrm{Na}-2 \mathrm{H}]^{-}$at $1318 \mathrm{~m} / z$. These ions were observed with all five matrices used herein. Additional analyte molecular ion peaks were detected using the cinnamic acid-based matrices SA and HCCA. Peaks at masses corresponding to what appears to be a cinnamic acid fragment were observed at $m / z 133$ and were unique to these compounds. Additional quasimolecular ions, albeit with lesser intensity, were detected at $1430 \mathrm{~m} / \mathrm{z}, 1452 \mathrm{~m} / \mathrm{z}$, as well as $1468 \mathrm{~m} / \mathrm{z}$ in the positive mode corresponding to cinnamic acid fragment adductions $[\mathrm{A}+\mathrm{X}]^{+}$, where $\mathrm{X}$ is likely the cinnamic acid fragment, and various species where the proton is exchanged by alkali metal ions including $[\mathrm{A}+\mathrm{X}+$ $\mathrm{Na}-\mathrm{H}]^{+}$and $[\mathrm{A}+\mathrm{X}+\mathrm{Na}+\mathrm{K}-2 \mathrm{H}]^{+}$. In the 


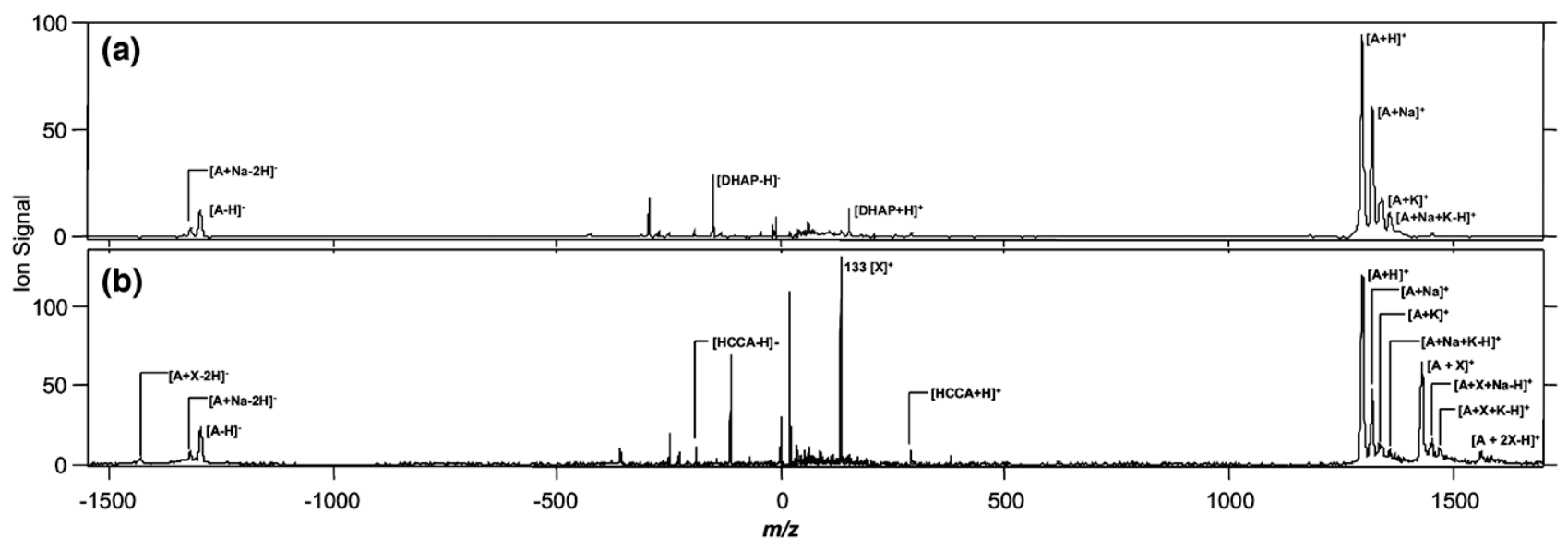

Figure 1. Spectra from single $\sim 1 \mu \mathrm{m}$ particles of angiotensin I (a), $1297 \mathrm{~m} / \mathrm{z}$, in the studied matrices (a) DHAP and (b) HCCA at a 100:1 matrix-to-analyte molar ratio. X denotes a cinnamic acid fragment. Pronounced angiotensin fragment peaks were not observed.

negative polarity, a doubly deprotonated but cinnamic acid fragment adducted ion was also observed at 1428 $\mathrm{m} / \mathrm{z}$. Due to the reduced analyte molecular ion signal when using FA as a matrix, cinnamic acid fragment adduct peaks were not visible, if present. Additionally, in contrast to previous work in aerosol mass spectrometry [34], solvent peaks were not observed.

\section{Aerodynamic Particle Diameter}

In 2005, Harris et al. reported that "the biomolecular ion signal from the polydisperse aerosol particles did not change as a function of size" [36]. This referred, in particular, to particles of bradykinin coated with picolinic acid, but the paper went on to more generally report that total ion signal in laser-ablation based aerosol mass spectrometry does not directly correlate with particle mass. They cited the 1996 work of Mansoori et al., in which the sodiated molecular ion of leucine enkephalin prepared in 3-nitrobenzyl alcohol did show such behavior for large particle sizes $(>4 \mu \mathrm{m})$, but in that same paper the ion signal appeared to fall for smaller particles $(2-3 \mu \mathrm{m})$ [35]. Observations in the present authors' laboratory suggested that size had some effect in the $\sim 0.5-2 \mu \mathrm{m}$ range, so more careful experiments were conducted.

An increase in particle aerodynamic diameter was correlated with an increase in angiotensin quasimolecular ion signal for all matrices studied at a 100:1 matrixto-analyte ratio. Aerodynamic diameter, $d_{a}$, is the diameter of a unit-density spherical particle having the same inertial properties in a gas as the particle of interest. It is defined as:

$$
d_{a}=d_{s} \sqrt{\rho}
$$

where $\rho$ is the particle's mass density, and $d_{s}$ is its Stokes diameter, which is equal to the physical diameter for spherical particles. The aerodynamic diameters gen- erated for all matrices ranged between $0.66 \mu \mathrm{m}$ and 1.70 $\mu \mathrm{m}$. For each matrix, the analyzed particles were separated into four size bins (0.66-0.92, 0.92-1.18, 1.18-1.44, and $1.44-1.70 \mu \mathrm{m})$. Average HCCA spectra from each size bin are shown in Figure 2. It is evident that an increase in particle size was accompanied by a significant increase in ion yield. Similar trends were observed for the other matrices. To quantify this trend, the average angiotensin cation peak area (incorporating all of the quasimolecular ions) was plotted against particle size for each of the matrices in Figure 3a.

To explain their observations, Harris et al. proposed a model in which the incident laser energy was internally focused in the aerosol particle. The resulting focal volume was the source of observed ions and was reported to be largely independent of particle size, thus explaining the constant ion yield [36]. Although this may be a valid model in some cases, it is inapplicable herein for several reasons. First, the present particles are not transparent. The solid-phase absorption coefficients at a wavelength of $355 \mathrm{~nm}$ are $1.4 \times 10^{5}, 1.3 \times 10^{5}, 1.0 \times$ $10^{5}$, and $1.4 \times 10^{5} \mathrm{~cm}^{-1}$ for FA, SA, DHB, and HCCA, respectively [44]. The absorption coefficient for DHAP was not found in the literature. Assuming that the absorption coefficients are not significantly reduced by the small amount of analyte present, this means that at least $65 \%$ of the incident energy is absorbed within the first $100 \mathrm{~nm}$ and $95 \%$ within the first $300 \mathrm{~nm}$ of the particles' surfaces. It is therefore improbable that any significant amount of energy would reach the theoretical focal volume. Second, for internal focusing to be highly effective, even in more transparent particles, the particles must be spherical and smooth. As typical SEM images show in Figure 4, the particles here are neither. Instead, the particles have rough, heterogeneous surfaces that vary significantly from one matrix to another.

In spite of the inapplicability of that model here, the underlying hypothesis that the volume in which energy is absorbed affects ion yield seems logical. Accurately 


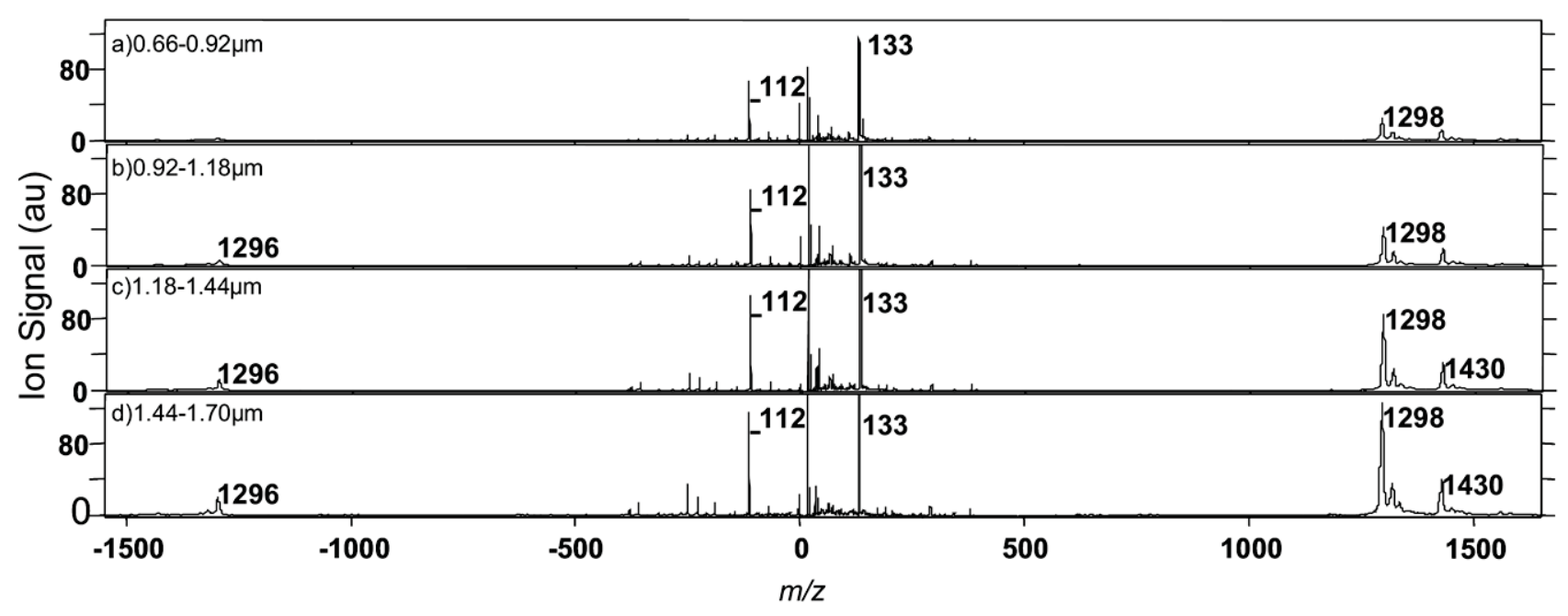

Figure 2. HCCA spectral averages separated according to the particle size (a) $0.66-0.92 \mu \mathrm{m}$, (b) $0.92-1.18 \mu \mathrm{m}$, (c) 1.18-1.44 $\mu \mathrm{m}$, and (d) $1.44-1.7 \mu \mathrm{m}$ show increased molecular analyte ion yield with increased particle size.

modeling the interaction of light with particles at this size scale, however, is difficult. Even Mie theory is only truly applicable to perfectly spherical particles. Furthermore, such calculations utilize the complex index of refraction, which is unknown for the matrices used. Nonetheless, even a greatly simplified treatment of laser-particle interaction, ignoring surface reflections, refraction, and various nonlinear effects, does produce informative results.

It is useful to define the absorption length, $L_{\alpha}$, as the inverse of the absorption coefficient (i.e., $1 / \alpha$ ). It has units of length and characterizes the distance that the laser light, and subsequently the laser energy, penetrates the particle; the shorter the absorption length, the more shallow the penetration. In a bulk sample, $95 \%$ of incident energy is absorbed within 3 absorption lengths of the surface. This suggests a rough definition for an effective absorption volume, $V_{u}$, within a particle of radius $R$, namely

$$
V_{a}=\pi R^{2} \times 3 L_{\alpha}-\pi \frac{9}{4} L_{\alpha}^{3}
$$

The first term is easily recognized as the particle cross section, $\pi R^{2}$, multiplied by 3 absorption lengths. The second term is a small correction, which accounts for light transmitted through the edges of the particle. Together, in this simplified treatment of laser-particle interaction, they represent an approximate volume in which most of the incident laser energy is absorbed.

This absorption volume was calculated for each particle in each size bin. The mean absorption volume was plotted against the mean molecular cation yield in Figure $3 \mathrm{~b}$ for the most absorbing matrix, HCCA, and the least absorbing matrix, DHB. The resulting curves are remarkably linear, although they do not pass perfectly through the origin. This lack of strict proportion- ality may be due, in part, to particle morphology and optical effects such as scattering and refraction. Other processes are almost certainly involved as well. Given the short absorption lengths, virtually all energy incident upon the particles is absorbed; consequently the absorbed energy varies roughly as $R^{2}$; (2) and Figure $3 \mathrm{~b}$ thus also imply that the molecular cation yield varied linearly with absorbed energy and surface area. This seems intuitively reasonable.

Surprisingly, few papers directly discuss the relationship between particle size and ion yield, but several have investigated the relationship between size and ablation efficiency [45] or size and laser-induced ion formation threshold [46, 47]. In general, ablation efficiency increases as particle size increases. The ion formation threshold is more variable but does decrease in some cases. This directly implies that at certain laser energies, for at least certain particle types, the ion yield per laser shot must increase with particle size. Consequently, these earlier findings corroborate and are at least qualitatively consistent with those presented herein. Ion yield is not universally independent of particle size.

A variation exists between this study and that presented previously by Harris et al. that should be noted. In that experiment, ions were produced and contained in a quadrupole ion trap. Data presented in a key figure seems to show that the ion capacity of the trap was often reached or exceeded even for small particles [36]. If true, increases in ion signal could easily have been obscured.

\section{Molar Ratio}

Sample solutions were prepared with a total concentration of $7.14 \times 10^{-4} \mathrm{M}$ and matrix-to-analyte molar ratios of $50 \mathrm{~K}: 1,10 \mathrm{~K}: 1,1 \mathrm{~K}: 1,100: 1$, and $10: 1$ to determine the 

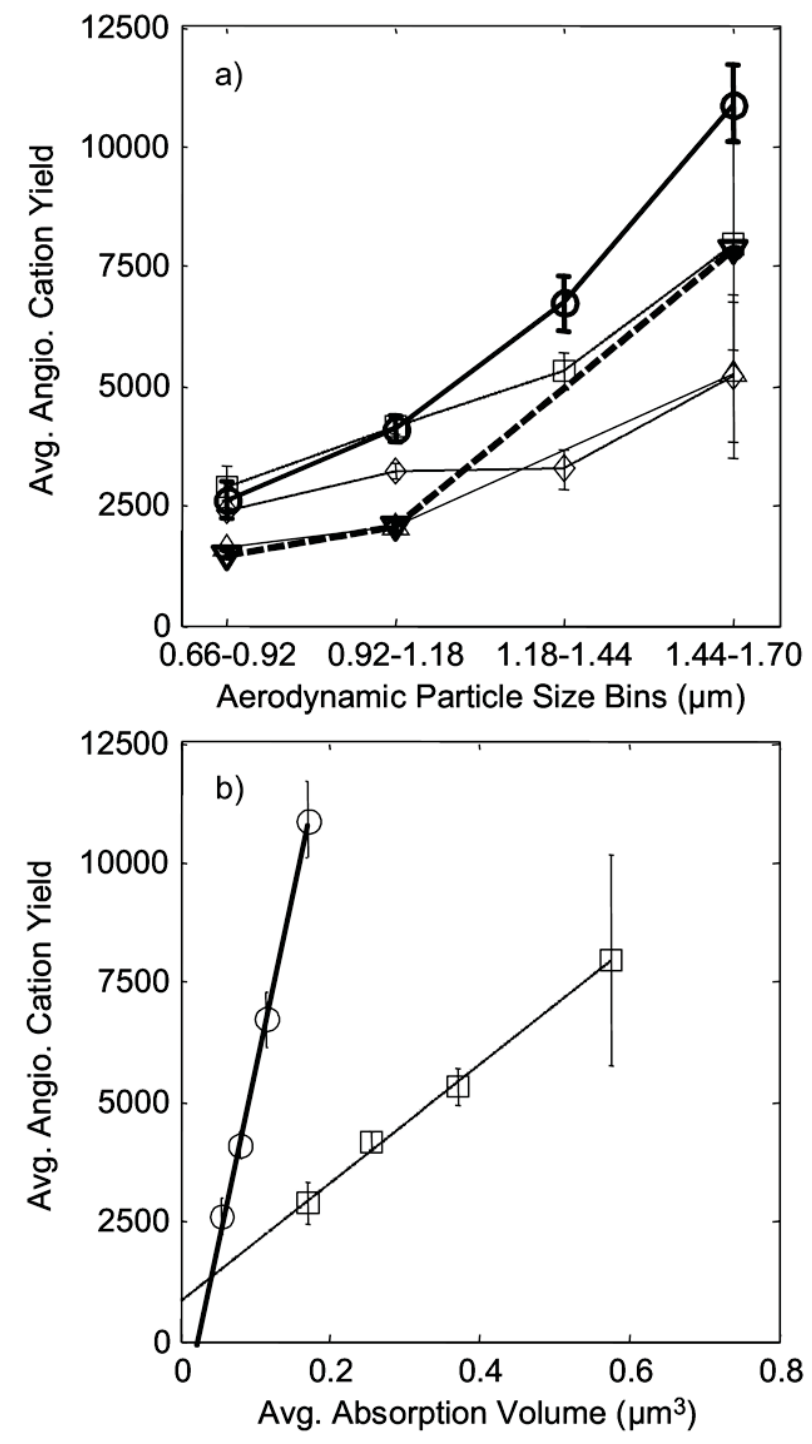

Figure 3. (a) The plot shows that the mean angiotensin cation yield increases with size for each matrix: HCCA (circle), DHAP (square), DHB (diamond), SA (triangle), and FA (inverted triangle). The vertical error bars represent the standard deviation of the mean. (b) The linear relationship between absorption volume and angiotensin cation yield is shown for the matrices HCCA (circle) and DHB (square).

optimal ratio yielding the most molecular analyte ions for a fixed particle size. For the largest molar ratios (50K:1 and $10 \mathrm{~K}: 1)$, no analyte ions were observed (Figure 5). The absence of analyte ions in the spectra was presumably due to the limited number of analyte molecules in the particles. Positive and negative analyte molecular ions were detected in the 1K:1, 100:1, and 10:1 ratios for all of the matrices. Analysis of the spectra in Figure 5 for DHB revealed that the $1 \mathrm{~K}: 1$ ratio yielded the highest ionization efficiency. The absolute analyte molecular ion yield in dual polarity, however, was found to be greatest with the 100:1 molar ratio for each matrix studied. This is not unreasonable, in spite of bulk MALDI results where ratios of $1 \mathrm{~K}: 1$ to $10 \mathrm{~K}: 1$ are often typical [48]. It is in fact consistent with the earlier single particle findings [27]. According to previous aerosol [33] and bulk [49, 50] MALDI studies, rapid crystallization enhances ion formation, thereby producing a lower optimal matrix-to-analyte ratio. Specifically, faster crystallization generates smaller crystals and facilitates greater contact between the matrix and analyte [27]. In the aerosolization system employed herein, a wet particle spends $\sim 1.6 \mathrm{~s}$ in the silica dryer; hence, this is the approximate time in which crystallization occurs. Additional drying and crystallization may occur in the transfer lines or upon introduction to vacuum, but the total crystallization time is still on the order of seconds. This is much faster than typical in bulk. The result, as shown in Figure 4, is an apparently homogenous particle with rough surface morphology. This surface varies with particle composition but in no case appears to be that of a well formed single crystal or even cluster of large crystals, which might be expected to result from slower crystallization. As will be discussed shortly, such a low matrix-to-analyte ratio is also favored by the unique plume dynamics of aerosol particles.

Results from the 10:1 M ratio studies showed both decreased analyte ion signal and reduced ionization efficiency. This is likely due, in part, to the analyte absorbing a greater fraction of the incident laser energy. If $\sigma_{\mathrm{A}}$ is the absorption cross section for the analyte, $\sigma_{\mathrm{M}}$ the absorption cross section for the matrix, and $\mathrm{R}$ the ratio of matrix to analyte, then a simple calculation suggests that the ratio of the energy absorbed by the analyte, $E_{a}$, to the total absorbed energy, $E_{t o t}$ is roughly

$$
\frac{E_{a}}{E_{\text {tot }}}=\frac{\sigma_{A}}{\sigma_{A}+R \sigma_{M}}
$$

Given that $\sigma_{A}$ is presumably comparable in magnitude to $\sigma_{M}$ or smaller, the analyte in particles with a 10:1 ratio absorb a factor of $\sim 10$ times more energy than in particles with a 100:1 ratio. Most of this is simply because there is more analyte, but the energy absorbed per analyte molecule does increase with decreasing $R$ as well. An additional factor is that the 10:1 ratio is likely too low to maximize the collision frequency between

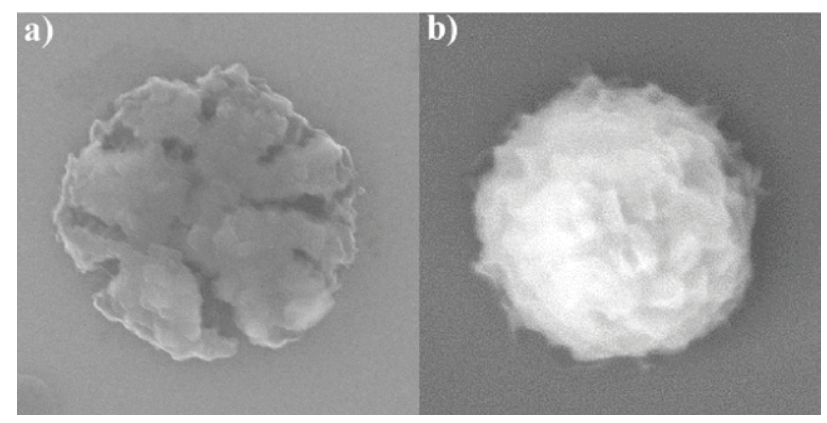

Figure 4. Select SEM images of single, $\sim 1 \mu \mathrm{m}$ particles of pure (a) HCCA and (b) SA. Rapid crystallization of the particles and the resulting surface morphology play potentially significant roles in the MALDI process. 


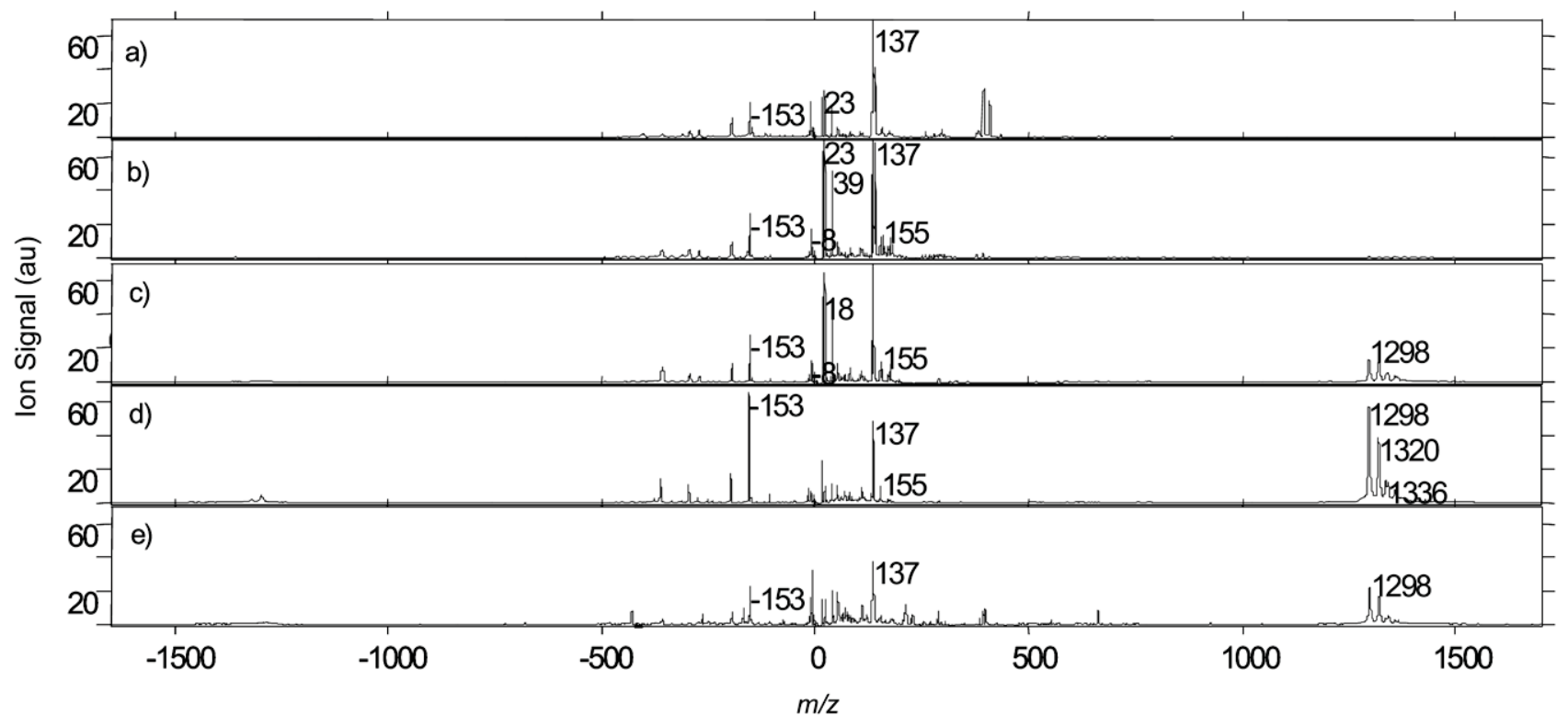

Figure 5. Spectral averages (150 particles each) of the matrix (DHB) and analyte (angiotensin) at various matrix-to-analyte molar ratios: (a) 50K:1, (b) 10K:1, (c) 1K:1, (d) 100:1, and (e) 10:1. The 100:1 matrix-to-analyte molar ratio yielded the greatest analyte ion signal in dual polarity, which is a much lower ratio than typically optimal in bulk.

analyte and matrix in the MALDI plume as will be shown.

A simple analysis of idealized bulk and particle MALDI experiments suggests that a lower matrix-toanalyte ratio is favored for particles. In the idealized bulk MALDI experiment, consider an infinite sheet of matrix and analyte with thickness $D$. In the idealized particle experiment, consider a cube of material (i.e., a particle) with the same thickness, $D$. Both samples have the same initial density, $\rho_{0}$. A laser with infinite diameter, zero pulse length, and finite fluence interacts with both samples, causing them to expand with velocity $v$ (in reality $v$ is expected to be somewhat different for the two cases but not by orders of magnitude). The density of the particle plume, $\rho_{\mathrm{p}}$, and the bulk plume, $\mu_{\mathrm{b}}$, as a function of time are consequently:

$$
\begin{aligned}
& \rho_{p}(t)=\rho_{0}\left(1+\frac{\nu}{D} t\right)^{-3} \\
& \rho_{b}(t)=\rho_{0}\left(1+\frac{\nu}{D} t\right)^{-1}
\end{aligned}
$$

The density of the particle, which is free to expand in three dimensions, drops much faster as time progresses than the density of the sheet, which is only free to expand in one dimension. This can be quantified in various ways, but the important conclusion is simply that there is much less time in the case of a particle for the critical collisions to occur between matrix and analyte that ultimately produce analyte ions. Consequently, this suggests that it is more important to maximize the collision rate in aerosol MALDI than in bulk MALDI. This does not mean that the collision rate is unimportant in bulk MALDI but simply that it is less important than in aerosol MALDI.

The collisions of most direct importance are those between analyte molecules and excited matrix molecules. A simple equation for the total collision rate per unit volume is:

$$
Z_{a \tilde{m}}=\sigma \nu \rho_{a} \rho_{\tilde{m}}
$$

where $\sigma$ is the collision cross section (not absorption cross section), $v$ is the mean relative velocity (which for the purposes of this simple model can essentially be considered the same as the $v$ mentioned above), $\rho_{a}$ is the analyte density and $\rho_{\tilde{m}}$ is the excited matrix density. In this case, $v$ is assumed fixed. Increasing $v$ does increase the collision rate, but it also increases the rate at which the density falls. It can be shown that these two effects cancel and $v$ ultimately has no effect upon the total number of collisions in this simple model. The collision rate is only effectively raised by maximizing the product of the two densities, which can be achieved in part by varying the matrix-to-analyte ratio, $R$. Before a specific value for $R$ can be obtained, however, a relationship between the number of excited matrix molecules, $N_{\tilde{m}}$, and the total number of (initially unexcited) matrix molecules, $N_{m}$, must be defined. A very general equation is simply $N_{\widetilde{m}}=c N_{m}^{n}$, where $c$ is essentially a proportionality constant and $n$ accounts for the fact that the excited matrix molecules of interest likely arise, at least in part, from the collisions of less excited matrix 
molecules. A simple derivation ultimately reveals that an optimal value of $R$ is:

$$
R=n \frac{V_{a}}{V_{m}} \approx n \frac{M_{a}}{M_{m}}
$$

where $V_{u}$ and $M_{a}$ are the volume and mass of a single analyte molecule, and $V_{m}$ and $M_{m}$ are the corresponding quantities for a matrix molecule. Given an analyte ten times the size of the matrix and a probable $n$ value of less than 3, the predicted optimal ratio of matrix-toanalyte is less than 30 . Many other factors influence the actual optimal ratio in practice, but the collision rate, which is of particular importance in aerosol MALDI, clearly favors a ratio far smaller than that typically used in bulk. This may, in part, explain the observations presented herein and those of others.

It should also be noted that $c$, the proportionality constant mentioned above, is actually a function of laser fluence that increases with fluence until fragmentation of the matrix inhibits creation of additional excited matrix molecules. Consequently, the collision rate increases with laser fluence over a significant range. Given the relative importance of the collision rate in aerosol MALDI compared to bulk, this potentially represents another factor contributing to the higher fluences found optimal.

\section{Matrix Selection}

Unlike most mass spectrometers, the BAMS system has the ability to obtain dual polarity mass spectra, making it ideal for simultaneous investigation of negative and positive ions created during a single MALDI event. Although absolute comparisons between the two polarities are not yet possible because of differing ion transmission efficiencies, relative comparisons can still be made. This is valuable when comparing the efficacy of different matrices. Indeed, the choice of matrix affects both the absolute and relative yields of positive and negative molecular analyte ions, as shown in Figure 6 for the 100:1 matrix-to-analyte molar ratio. Such particle by particle comparisons cannot be made with a single polarity instrument, although various effects of matrix selection have been observed [27].

As previously stated, the total angiotensin cation and anion peak areas include all positive and negative quasimolecular ions, respectively. The analyte molecular ion yield increased in both polarities in the order of FA, SA, DHAP, DHB, and HCCA. Note that a single laser fluence $\left(260 \mathrm{~mJ} / \mathrm{cm}^{2}\right)$, optimized for SA, was used. A positive correlation between total positive and total negative analyte molecular ion yield was observed for all of the matrices. The proportionality constant or slope, as illustrated and labeled in Figure 6, is matrix dependent, the steeper the slope, the greater the relative preference in anion production. On average, the angiotensin ion yield in the positive polarity is 10-fold greater than in the negative, which is larger than the expected

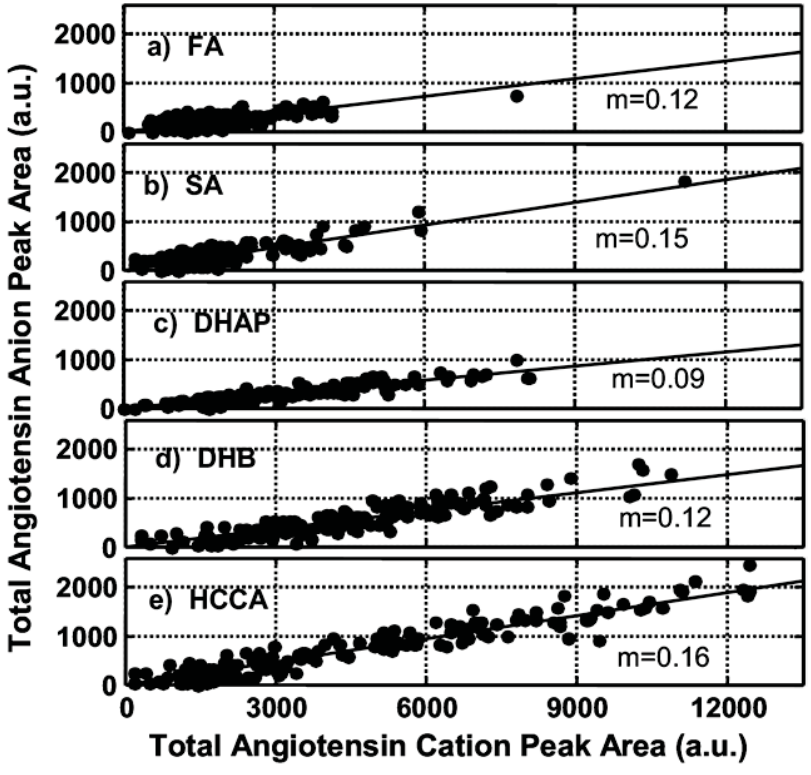

Figure 6. Total angiotensin anion peak area versus cation peak area for the matrices (a) FA, (b) SA, (c) DHAP, (d) DHB, and (e) HCCA. The positive and negative total analyte ion yields are positively correlated for all of the matrices. The slope of the fit line, $\mathrm{m}$, varies by matrix with HCCA having the greatest relative preference for angiotensin anion to angiotensin cation production and the highest absolute yield of both.

difference in ion transmission efficiencies. A quantitative model explaining these observations, however, does not yet exist.

The apparent disparity between polarities and the variations between matrices may be explained, in part, by the matrix dependent production of free electrons. Free electrons directly limit the production of negative analyte molecular ions since charge is strictly conserved in single particle MALDI. This is unusual since most bulk MALDI experiments are performed on a metal sample plate, which can potentially act as either a source or sink of charge. Frankevich et al., for example, clearly showed that free electrons could be generated from the organic/metal interface [51]. Apart from charge conservation, they further showed that free electrons participate in the formation of negative matrix ions, which subsequently suppress positive analyte signal.

There are, of course, many other processes that contribute to the pattern of ions observed, and many of these are matrix dependent. With the exception of FA, the most dominant molecular ion peak present for the matrices investigated was the protonated angiotensin ion. As such, the matrix proton affinity (PA) is a potentially relevant quantity. A rough negative correlation is in fact found between matrix proton affinity and protonated angiotensin ion yield. The ion yield must ultimately be constrained by the amount of energy absorbed. It is interesting to note therefore that the solid-phase absorption coefficients, listed in the particle size section, are not well correlated with the observed 
yields. This unintuitive result, however, can largely be explained. Even in the case of DHB, which absorbs most weakly, the majority of the incident laser energy is absorbed within the first $100 \mathrm{~nm}$ of the particle surface. Very little light or energy is transmitted through any of the particles. All particles of a given size thus absorb nearly the same amount of energy $(\sim 2 \mathrm{~nJ}$ for a $1 \mu \mathrm{m}$ particle), regardless of the absorption coefficient or matrix.

Although the amount of energy absorbed by a particle is largely independent of matrix type, the matrixdependent particle morphology likely plays a significant role in determining where the energy is absorbed, and this may be significant. As shown in Figure 4, single matrix particles vary in texture and morphology, thereby influencing the overall surface area and access to the particle core. For instance, the greater number of fissures and cracks in the HCCA particles increases the overall surface area and may allow the DI laser to penetrate more deeply into the particle. The addition of analyte at varying ratios could cause further morphological changes. The quantitative effects of laser interactions with these features are unknown.

\section{Conclusions}

Parameters that influence molecular analyte ion yield were examined by studying single aerosol MALDI particles. As expected, a relatively high fluence was required to maximize the ion signal. In contrast to earlier findings, increased aerodynamic particle size was clearly correlated with increased analyte ion signal. The signal appeared to vary linearly with absorption volume, absorbed energy, and surface area, which is intuitively reasonable. The molar ratio yielding optimal ion signal (100:1) in aerosol MALDI was approximately two orders of magnitude lower than typical in bulk MALDI. This low ratio may be due in part to the rapid decrease in particle plume density, which makes the collision rate within the plume a more critical factor. The optimal matrix, based on the above findings, was HCCA. A clear path for future studies is to progressively consider larger proteins and even toxins (or their stimulants), viruses, spores, and cells. Ultimately, such studies should enable more sensitive and reliable bioaerosol detection.

\section{Acknowledgments}

The authors acknowledge that this work was performed under the auspices of the U.S. Department of Energy by the University of California, Lawrence Livermore National Laboratory under Contract W-7405-Eng-48. They gratefully acknowledge support for this project by the LLNL Lab-directed research and development (LDRD) program under project numbers 05-ERD-053 and NSFCHE-0503765. Additionally, ELM thanks the National Institute of Health, the Lawrence Livermore National Laboratory Student Employee Graduate Research Fellowship Program, the Alfred P. Sloan Foundation, and the UNCF-Merck Science Initiative Program for providing research funding.

\section{References}

1. Tobias, H. J.; Schafer, M. P.; Pitesky, M.; Fergenson, D. P.; Horn, J.; Frank, M.; Gard, E. E. Bioaerosol Mass Spectrometry for Rapid Detection of Individual Airborne Mycobacterium tuberculosis H37Ra Particles. Appl. Environ. Microbiol. 2005, 71(10), 6086-6095.

2. McJimpsey, E. L.; Steele, P. T.; Coffee, K. R.; Fergenson, D. P.; Riot, V. J.; Woods, B. W.; Gard, E. E.; Frank, M.; Tobias, H. J.; Lebrilla, C. In Detection of Biological Particles in Ambient Air Using Bioaerosol Mass Spectrometry; Chemical and Biological Sensing VII: Orlando (Kissimmee), FL, 2006/05/05/; Gardner, P. J.; Fountain , A. W. III, Eds.; SPIE: Orlando (Kissimmee), FL, 2006; pp 62180B-62190B.

3. Steele, P. T.; McJimpsey, E. L.; Coffee, K. R.; Fergenson, D. P.; Riot, V. J.; Tobias, H. J.; Woods, B. W.; Gard, E. E.; Frank, M. In Characterization of Ambient Aerosols at the San Francisco International Airport Using Bioaerosol Mass Spectrometry; Chemical and Biological Sensing VII: Orlando (Kissimmee), FL, 2006/05/05/; Gardner, P. J.; Fountain, A. W. III, Eds.; SPIE: Orlando (Kissimmee), FL, 2006; pp 62180A-62189A.

4. Czerwieniec, G. A.; Russell, S. C.; Tobias, H. J.; Pitesky, M. E.; Fergenson, D. P.; Steele, P.; Srivastava, A.; Horn, J. M.; Frank, M.; Gard, E. E.; Lebrilla, C. B. Stable Isotope Labeling of Entire Bacillus atrophaeus Spores and Vegetative Cells Using Bioaerosol Mass Spectrometry. Anal. Chem. 2005, 77(4), 1081-1087.

5. Fergenson, D. P.; Pitesky, M. E.; Tobias, H. J.; Steele, P. T.; Czerwieniec, G. A.; Russell, S. C.; Lebrilla, C. B.; Horn, J. M.; Coffee, K. R.; Srivastava, A.; Pillai, S. P.; Shih, M. T. P.; Hall, H. L.; Ramponi, A. J.; Chang, J. T.; Langlois, R. G.; Estacio, P. L.; Hadley, R. T.; Frank, M.; Gard, E. E. Reagentless Detection and Classification of Individual Bioaerosol Particles in Seconds. Anal. Chem. 2004, 76(2), 373-378.

6. Srivastava, A.; Pitesky, M. E.; Steele, P. T.; Tobias, H. J.; Fergenson, D. P. Horn, J. M.; Russell, S. C.; Czerwieniec, G. A.; Lebrilla, C. S.; Gard, E. E.; Frank, M. Comprehensive Assignment of Mass Spectral Signatures from Individual Bacillus atrophaeus Spores in Matrix-Free Laser Desorption/Ionization Bioaerosol Mass Spectrometry. Anal. Chem. 2005, 77(10), 3315-3323.

7. Sinha, M. P.; Platz, R. M.; Vilker, V. L.; Friedlander, S. K. Analysis of Individual Biological Particles by Mass Spectrometry. Int. J. Mass Spectrom. Ion Processe. 1984, 57(1), 125-133.

8. Marijnissen, J.; Scarlett, B.; Verheijen, P. Proposed Online Aerosol Analysis Combining Size Determination, Laser-Induced Fragmentation and Time-of-Flight Mass Spectroscopy. J. Aerosol Sci. 1988, 19(7), 13071310.

9. McKeown, P. J.; Johnston, M. V.; Murphy, D. M. Online Single-Particle Analysis by Laser Desorption Mass Spectrometry. Anal. Chem. 1991, 63(18), 2069-2073.

10. Noble, C. A.; Nordmeyer, T.; Salt, K.; Morrical, B.; Prather, K. A. Aerosol Characterization Using Mass Spectrometry. Trac-Trends Anal. Chem. 1994, 13(5), 218-222.

11. Hinz, K. P.; Kaufmann, R.; Spengler, B. Simultaneous Detection of Positive and Negative Ions from Single Airborne Particles by Real-Time Laser Mass Spectrometry. Aerosol Sci. Technol. 1996, 24(4), 233-242.

12. Gard, E.; Mayer, J. E.; Morrical, B. D.; Dienes, T.; Fergenson, D. P.; Prather, K. A. Real-Time Analysis of Individual Atmospheric Aerosol Particles: Design and Performance of a Portable ATOFMS. Anal. Chem. 1997, 69(20), 4083-4091.

13. Steele, P. T.; Tobias, H. J.; Fergenson, D. P.; Pitesky, M. E.; Horn, J. M.; Czerwieniec, G. A.; Russell, S. C.; Lebrilla, C. B.; Gard, E. E.; Frank, M. Laser Power Dependence of Mass Spectral Signatures from Individual Bacterial Spores in Bioaerosol Mass Spectrometry. Anal. Chem. 2003, 75(20), 5480-5487.

14. Dale, J. M.; Yang, M.; Whitten, W. B.; Ramsey, J. M. Chemical Characterization of Single Particles by Laser Ablation/Desorption in a Quadrupole Ion-Trap Mass-Spectrometer. Anal. Chem. 1994, 66(20), 34313435 .

15. Thomson, D. S.; Murphy, D. M. Analyzing Single Aerosol-Particles in Real-Time-Composition Analysis of Aerosols is Critical to an Understanding of Their Origins and Fate-This is Done by Combining Time-of-Flight Mass Spectroscopy with Optical-Particle Detections and Sizing. CHEMTEC. 1994, 24(12), 30-35.

16. Murphy, D. M.; Thomson, D. S. Laser Ionization Mass Spectroscopy of Single Aerosol Particles. Aerosol Sci. Technol. 1995, 22(3), 237-249.

17. Murphy, D. M.; Thomson, D. S.; Mahoney, T. M. J. In Situ Measurements of Organics, Meteoritic Material, Mercury, and Other Elements in Aerosols at 5 to 19 Kilometers. Scienc. 1998, 282(5394), 1664-1669.

18. Gieray, R. A.; Reilly, P. T. A.; Yang, M.; Whitten, W. B.; Ramsey, J. M. Real-Time Detection of Individual Airborne Bacteria. J. Microbiol. Method. 1997, 29(3), 191-199.

19. Yang, M.; Reilly, P. T. A.; Gieray, R. A.; Whitten, W. B.; Ramsey, J. M. Complete Chemical Analysis of Aerosol Particles in Real-Time. J. Korean Phys. Soc. 1997, 30(2), 359-363.

20. Nash, D. G.; Baer, T.; Johnston, M. V. Aerosol mass spectrometry: An introductory review. Int. J. Mass Spectrom. 2006, 258(1/3), 2-12.

21. Suess, D. T.; Prather, K. A. Mass Spectrometry of Aerosols. Chem. Rev. 1999, 99(10), 3007-3035.

22. Karas, M.; Bachmann, D.; Hillenkamp, F. Influence of the Wavelength in High-Irradiance Ultraviolet-Laser Desorption Mass Spectrometry of Organic Molecules. Anal. Chem. 1985, 57(14), 2935-2939. 
23. Karas, M.; Hillenkamp, F. Laser Desorption Ionization of Proteins with Molecular Masses Exceeding 10000 Daltons. Anal. Chem. 1988, 60(20), 2299-2301.

24. Tanaka, K.; Ido, Y.: Akita, S.; Yoshida, Y.; Yoshida T. Detection of High Mass Molecules by Laser Desorption Time-of-Flight Mass Spectrometry. Proceedings of the Second Japan-China Joint Symposium on Mass Spectrometry; Kyoto, Japan, May 1987.

25. Hathout, Y.; Setlow, B.; Cabrera-Martinez, R. M.; Fenselau, C.; Setlow, P. Small, Acid-Soluble Proteins as Biomarkers in Mass Spectrometry Analysis of Bacillus spores. Appl. Environ. Microbiol. 2003, 69(2), 11001107.

26. Murray, K. K.; Russell, D. H. Liquid Sample Introduction for MatrixAssisted Laser-Desorption Ionization. Anal. Chem. 1993, 65(18), $2534-$ 2537.

27. Beeson, M. D. Murray, K. K. Russell, D. H. Aerosol Matrix-Assisted Laser-Desorption Ionization-Effects of Analyte Concentration and Matrix-to-Analyte Ratio. Anal. Chem. 1995, 67(13), 1981-1986.

28. Fei, X.; Wei, G.; Murray, K. K. Aerosol MALDI with a Reflectron Time-of-Flight Mass Spectrometer. Anal. Chem. 1996, 68(7), 1143-1147.

29. He, L.; Murray, K. K. A Laminar Flow Nebulizer for Aerosol MALDI. Anal. Chem. 1997, 69(17), 3613-3616.

30. He, L.; Murray, K. K. Three hundred thirty-seven nm Matrix-Assisted Laser Desorption/Ionization of Single Aerosol Particles. J. Mass Spectrom. 1999, 34(9), 909-914.

31. He, L.; Wei, G.; Murray, K. K. Fragmentation of Vitamin B-12 in Aerosol Matrix-Assisted Laser Desorption Ionization. J. Am. Soc. Mass Spectrom. 1997, 8(2), 140-147.

32. Jackson, S. N.; Murray, K. K. Matrix Addition by Condensation for Matrix-Assisted Laser Desorption/Ionization of Collected Aerosol Particles. Anal. Chem. 2002, 74(18), 4841-4844.

33. Murray, K. K.; Russell, D. H. Aerosol Matrix-Assisted Laser-Desorption Ionization Mass Spectrometry. J. Am. Soc. Mass Spectrom. 1994, 5(1), 1-9.

34. Russell, D. H.; Beeson, M. D. Aerosol Matrix-Assisted Laser Desorption Ionization Mass Spectrometry. I. Effect of Solvent on Ion Signal. J. Mass Spectrom. 1996, 31(3), 295-302

35. Mansoori, B. A.; Johnston, M. V.; Wexler, A. S. Matrix-Assisted Laser Desorption/Ionization of Size- and Composition-Selected Aerosol Particles. Anal. Chem. 1996, 68(20), 3595-3601.

36. Harris, W. A.; Reilly, P. T. A.; Whitten, W. B. MALDI of Individual Biomolecule-Containing Airborne Particles in an Ion Trap Mass Spectrometer. Anal. Chem. 2005, 77(13), 4042-4050.

37. Stowers, M. A.; van Wuijckhuijse, A. L.; Marijnissen, J. C. M.; Scarlett B.; van Baar, B. L. M.; Kientz, C. E. Application of Matrix-Assisted Laser Desorption/Ionization to On-Line Aerosol Time-of-Flight Mass Spectrometry. Rapid Commun. Mass Spectrom. 2000, 14(10), 829-833.

38. van Wuijckhuijse, A. L.; Stowers, M. A.; Kleefsman, W. A.; van Baar, B. L. M.; Kientz, C. E.; Marijnissen, J. C. M. Matrix-Assisted Laser Desorption/Ionization Aerosol Time-of-Flight Mass Spectrometry for the Analysis of Bioaerosols: Development of a Fast Detector for Airborne Biological Pathogens. J. Aerosol Sci. 2005, 36(5/6), 677-687.

39. Russell, S. C.; Czerwieniec, G.; Lebrilla, C.; Steele, P.; Riot, V.; Coffee, K. Frank, M.; Gard, E. E. Achieving High Detection Sensitivity (14 zmol) of Biomolecular Ions in Bioaerosol Mass Spectrometry. Anal. Chem. 2005, 77(15), 4734-4741.

40. Czerwieniec, G. A.; Russell, S. C.; Lebrilla, C. B.; Coffee, K. R.; Riot, V. Steele, P. T.; Frank, M.; Gard, E. E. Improved Sensitivity and Mass Range in Time-of-Flight bioaerosol Mass Spectrometry Using an Electrostatic Ion Guide. J. Am. Soc. Mass Spectrom. 2005, 16(11), 1866-1875.

41. Steele, P. T.; Srivastava, A.; Pitesky, M. E.; Fergenson, D. P.; Tobias, H. J.; Gard, E. E.; Frank, M. Desorption/Ionization Fluence Thresholds and Improved Mass Spectral Consistency Measured Using a Flattop Laser Profile in the Bioaerosol Mass Spectrometry of single Bacillus endospores. Anal. Chem. 2005, 77(22), 7448-7454

42. Dreisewerd, K.; Schurenberg, M.; Karas, M.; Hillenkamp, F. Influence of the Laser Intensity and Spot Size on the Desorption of Molecules and Ions in Matrix-Assisted Laser-Desorption Ionization with a Uniform Beam Profile. Int. J. Mass Spectrom. Ion Processe. 1995, 141(2), 127-148.

43. Wenzel, R. J.; Prather, K. A. Improvements in Ion Signal Reproducibility Obtained Using a Homogeneous Laser Beam for On-Line Laser Desorption/Ionization of Single Particles. Rapid Commun. Mass Spectrom. 2004 18(13), 1525-1533.

44. Allwood, D. A.; Dreyfus, R. W.; Perera, I. K.; Dyer, P. E. Optica Absorption of Matrix Compounds for Laser-Induced Desorption and Ionization (MALDI). Appl. Surf. Sci. 1997, 109/110 154-157.

45. Kane, D. B.; Johnston, M. V. Size and Composition Biases on the Detection of Individual Ultrafine Particles by Aerosol Mass Spectrometry. Environ. Sci. Technol. 2000, 34(23), 4887-4893.

46. Thomson, D. S.; Middlebrook, A. M.; Murphy, D. M. Thresholds for Laser-Induced Ion Formation from Aerosols in a Vacuum Using Ultraviolet and Vacuum-Ultraviolet Laser Wavelengths. Aerosol Sci. Technol. 1997, 26(6), 544-559.

47. Thomson, D. S.; Murphy, D. M. Laser-Induced Ion Formation Thresholds of Aerosol-Particles in a Vacuum. Appl. Opt. 1993, 32(33), 68186826.

48. Strupat, K.; Karas, M.; Hillenkamp, F. 2,5-Dihydroxybenzoic AcidNew Matrix for Laser Desorption Ionization Mass Spectrometry. Int. J. Mass Spectrom. Ion Processe. 1991, 111, 89-102.

49. Xiang, F.; Beavis, R. C. A Method to Increase Contaminant Tolerance in Protein Matrix-Assisted Laser-Desorption Ionization by the Fabrication of Thin Protein-Doped Polycrystalline Films. Rapid Commun. Mass Spectrom. 1994, 8(2), 199-204.

50. Cohen, S. L.; Chait, B. T. Influence of Matrix Solution Conditions on the MALDI-MS Analysis of Peptides and Proteins. Anal. Chem. 1996, 68(1) 31-37.

51. Frankevich, V. E.; Zhang, J.; Friess, S. D.; Dashtiev, M.; Zenobi, R. Role of electrons in laser desorption/ionization mass spectrometry. Anal. Chem. 2003, 75(22), 6063-6067. 